\title{
Caracterização de associação entre a sazonalidade de larvas de Dermatobia hominis em bovinos, dípteros potenciais vetores e dados meteorológicos de três diferentes locais no Rio de Janeiro
}

\author{
Characterization of association between seasonality of larvaes of \\ Dermatobia hominis in bovines, potentials vectors dipterans and \\ meteorological data of three different places in Rio de Janeiro
}

Fábio Silva de Souza, ${ }^{*}$ Maria Clara da Silva Negreiros Botelho, ${ }^{* \star}$ Raquel Silva Lisbôa***

\begin{abstract}
Resumo
O presente estudo observacional teve por objetivo caracterizar a associação entre a sazonalidade das larvas de Dermatobia hominis em bovinos, ocorrência de dípteros potenciais vetores e dados meteorológicos. Realizaram-se contagens mensais das larvas de $D$. hominis em dez bovinos de três propriedades rurais (A, B e C) durante os anos de 2006 e 2007 e capturas de dípteros utilizando armadilhas Adultrap ${ }^{\circledR}$, tendo como isca sardinha em decomposição. Obtiveram-se os dados climáticos de temperatura, precipitação pluvial e umidade relativa dos municípios abrangidos. Aplicou-se o Teste de Spearman para verificar as associações propostas. Ocorreu infestação por bernes durante todo o estudo nas três propriedades e, associação $\left(r_{s}=0,63\right)$ entre os resultados de flutuação de bernes da propriedade $\mathrm{A}$ com os dados climáticos de umidade relativa para o ano de 2006. Houve correlação entre os valores totais de dípteros coletados na propriedade $B$, com a precipitação pluvial $\left(r_{s}=0,80\right)$ e na propriedade C com a temperatura $\left(r_{s}=0,60\right)$, ambas no ano de 2007. Constatou-se uma falta de padrão na associação entre as flutuações mensais e por períodos, chuvoso e seco, das larvas de $D$. hominis com as flutuações de dípteros potenciais vetores e de ambas com os dados meteorológicos.
\end{abstract}

Palavras-chave: moscas, fatores climáticos, sazonalidade.

\begin{abstract}
The objective of the present observational study was to characterize the association between the seasonality of Dermatobia hominis larvae in bovines, potentials vectors dipterans occurrence and the meteorological data. Monthly counts of $D$. hominis larvae in ten bovines of three rural properties (A, B and C) during the years of 2006 and 2007 were developed and, capture of dipterans was realized using three snares Adultrap ${ }^{\circledR}$ having sardine in decomposition as bait. Climatic data of temperature, pluvial precipitation and relative humidity of the embraced municipalities were obtained. Spearman test was applied to verify the associations proposed. It was happened infestation with $D$. hominis larvae along all the study in the three properties and, association $\left(r_{s}=0.63\right)$ among the results of larvae fluctuation of the property A with the climatic data of relative humidity for the year of 2006. There was correlation among total values of dipterans collected in B property, with the pluvial precipitation $\left(r_{s}=0.80\right)$ and in $C$ property with the temperature $\left(r_{s}=0.60\right)$, both in 2007. An absence of standard was evidenced in the association between monthly fluctuations and for periods, rainy and dry, of $D$. hominis larvae with fluctuations of potentials dipterans vectors and of both with meteorological data.
\end{abstract}

Keywords: flies, climatic factors, seasonality.

\section{Introdução}

A distribuição sazonal das larvas de Dermatobia hominis (bernes) pode variar em função de um conjunto de diferentes condições geográficas e bióticas em cada região. Em um inquérito nacional sobre infestações de carrapato, míase e berne realizado por Horn e Antônio (1983), onde foram apontadas as regiões do país com maiores incidências registradas de berne, aparecendo em primeiro lugar a Região Sudeste, a Região Centro-Oeste em segundo e a Região Sul em terceiro, sendo as regiões Norte e Nordeste as menos infestadas. Observando-se cada região, verifica-se que apresentam características peculiares que facilitam ou

\footnotetext{
* Fundação Carlos Chagas Filho de Amparo à Pesquisa do Estado do Rio de Janeiro (FAPERJ) - Universidade Federal Rural do Rio de Janeiro (UFRRJ). Rua Nove, 95, Vila Operária - Nova Iguaçu, RJ - CEP 26015-260. A quem enviar a correspondência: E-mail: souzamedvet@yahoo.com.br

** Curso de Pós-Graduação em Ciências Veterinárias, do Instituto de Veterinária (IV), UFRRJ. mariaclarabotelho@yahoo.com.br;

*** Curso de Pós-Graduação em Ciências Veterinárias, IV, UFRRJ. raquel.silvalisboa@gmail.com
} 
dificultam a distribuição da dermatobiose, principalmente em bovinos.

O objetivo do presente estudo foi avaliar a associação entre a sazonalidade da dermatobiose em bovinos, a ocorrência de dípteros potenciais vetores e os fatores climáticos, temperatura, precipitação pluvial e umidade relativa.

\section{Material e métodos}

\section{Locais de estudo}

Os dados para avaliação da sazonalidade de larvas de $D$. hominis foram coletados no período de janeiro de 2006 a dezembro de 2007, em três propriedades rurais denominadas A, B e C, todas no estado do Rio de Janeiro.

$A$ propriedade $A$ refere-se à Fazendinha Agroecológica, pertencente ao convênio EMBRAPA/Pesagro-RJ/UFRRJ, destinada ao Sistema Integrado de Produção Agroecológica. Estava localizada no município de Seropédica na latitude $22^{\circ} 45^{\prime} 20.63^{\prime \prime S}$ e longitude $43^{\circ} 40^{\prime} 25.29 " \mathrm{O}$, com variações altimétricas entre 0 e 80 metros. 0 sistema instituído era o de Produção Orgânica, contando com diferentes meios de cultivo, criações de aves, além da produção de leite de vaca.

A propriedade $B$ encontrava-se próxima à área urbana, no bairro Guarajuba, no município de Paracambi, na latitude $22^{\circ} 38^{\prime} 2.79 " S$ e longitude $43^{\circ} 43^{\prime} 11.01^{\prime \prime} \mathrm{O}$, sendo constituída basicamente por pastagem nativa e vegetação arbustiva principalmente nos pequenos vales formados entre as pequenas elevações montanhosas. As variações altimétricas também alternavam-se entre 0 e 80 metros e as declividades entre 0 e 2,5\%. Possuía animais de diferentes espécies como equídeos utilizados para transporte, cães, aves e uma criação de suínos, além de um rebanho bovino composto por aproximadamente 65 animais mestiços. A produção de produção de leite era pequena, voltada para atender apenas o consumo local, com a ordenha de 13 vacas apenas.

A propriedade $C$ ficava no bairro lbituporanga, $3^{\circ}$ Distrito do município de Itaguaí, na latitude $22^{\circ} 43^{\prime} 53.21$ "S e longitude $43^{\circ} 47^{\prime} 56.07 " 0$. A área desta propriedade apresenta variações na altitude do relevo entre 40 e 200 metros e declividades entre 2,5 e $20 \%$. Além do rebanho bovino, possuía equídeos utilizados para transporte e cães sendo todos os animais mestiços e a produção de leite era direcionada para elaboração de laticínios, principalmente queijo minas frescal.

As atividades de laboratório foram realizadas no Laboratório de Miíases Tropicais (LMT) do Departamento de Parasitologia na Universidade Federal Rural do Rio de Janeiro.

\section{Avaliação da variação sazonal de bernes}

Foram efetuadas contagens mensais de larvas de $D$. hominis em dez animais de cada propriedade, por inspeção visual e, quando necessário, palpação ou compressão dos nódulos. A contagem foi realizada em todo o corpo de cada animal e foram calculadas as médias mensais a partir dos dados obtidos por animal. Na propriedade A, utilizou-se tronco de contenção para a realização das contagens e nas propriedades $\mathrm{B}$ e $\mathrm{C}$, realizadas com os animais soltos no curral. A faixa etária de todos os animais utilizados nas contagens variou entre três e sete anos.

\section{Coleta de dípteros potenciais vetores}

A avaliação da sazonalidade de dípteros muscoides ocorreu por meio de coletas mensais e simultâneas, nas três propriedades rurais selecionadas. Em cada propriedade foi colocada, mensalmente, uma armadilha Adultrap ${ }^{\circledR}$ (Souza et al., 2009). As armadilhas permaneciam penduradas a um metro do solo em um ponto livre da interferência direta de humanos e de animais, tendo como isca duas sardinhas com aproximadamente $70 \mathrm{~g}$ cada, retiradas do congelador e mantidas a temperatura ambiente por 24 horas antes de serem introduzidas na armadilha. Após 48 horas, as armadilhas eram recolhidas, rotuladas, acondicionadas em sacos plásticos e transportadas ao LMT, onde eram acomodadas em congelador a $-18^{\circ} \mathrm{C}$ por 36 horas. Após este tempo os insetos eram retirados e transferidos para frasco rotulado e retornados ao congelador. Para a identificação dos insetos, estes eram descongelados e secos em temperatura ambiente, examinados com auxílio de lupa estereoscópica e classificados com auxílio de chave taxonômica para famílias de dípteros segundo Guimarães e Papavero (1999) e Serra-Freire e Mello (2006).

\section{Dados climáticos}

Os dados dos fatores climáticos, temperatura média compensada $\left({ }^{\circ} \mathrm{C}\right)$, precipitação pluvial total $(\mathrm{mm})$ e umidade relativa (\%) relativos às Normais Climatológicas foram obtidos dos dados publicados pelo Departamento Nacional de Meteorologia (D.N.M., 1992), e os dados referentes aos anos de 2006 e 2007 foram cedidos pela Estação Ecologia

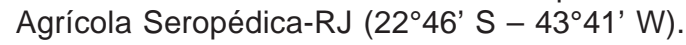

\section{Análise estatística}

O coeficiente de correlação para postos de Spearman $\left(r_{s}\right)$ foi utilizado para verificar a existência de correlação entre os dados climáticos mensais registrados nos anos de 2006, 2007 e das Normais Climatológicas; o número médio de bernes e os dados climáticos mensais; entre o número médio de bernes e o total de dípteros coletados e por famílias.

Os Testes de Kruskal-Wallis e Mann-Whitney foram empregados na verificação de diferenças significativas entre os períodos chuvoso e seco, por propriedade, para a média mensal de bernes.

\section{Resultados e discussão}

Os dados dos fatores climáticos: temperatura média compensada $\left({ }^{\circ} \mathrm{C}\right)$, precipitação pluvial total $(\mathrm{mm})$, umidade relativa (\%) dos anos de 2006, 2007 e das Normais Climatológicas, estão no Tabela 1. Os valores registrados para estes fatores estão dentro das faixas consideradas como adequadas à ocorrência da dermatobiose em bovinos (Moya Borja, 1966; Guimarães e Papavero, 1999).

As temperaturas médias compensadas mensais entre os anos de 2006 e 2007 estão correlacionadas entre si $\left(r_{s}=0,95\right.$, $p=0,0001)$. Os dados para este fator do ano de 2006 e 2007 com as Normais $\left(r_{s}=0,96, p=0,0001 ; r_{s}=0,90, p=0,0002\right.$, respectivamente) caracterizando a regularidade deste parâmetro durante o período do estudo. Entretanto, o fator precipitação pluvial total mensal esteve correlacionada entre 
Tabela 1: Médias mensais da temperatura média compensada $\left({ }^{\circ} \mathrm{C}\right)$, da precipitação pluvial total $(\mathrm{mm})$, da umidade relativa (\%) dos anos de 2006, 2007 e Normais Climatológicas (1961-1990), Estação Ecologia Agrícola Seropédica-RJ

\begin{tabular}{lccccccccc}
\hline \multicolumn{3}{c}{ Meses } & \multicolumn{3}{c}{ Temperatura média } & \multicolumn{3}{c}{ Precipitação total $(\mathrm{mm})$} & \multicolumn{3}{c}{ Umidade relativa (\%) } \\
& 2006 & 2007 & $\begin{array}{c}\left.{ }^{\circ} \mathrm{C}\right) \\
\text { Nor }\end{array}$ & 2006 & 2007 & Normais & 2006 & 2007 & Normais \\
\hline Jan & 25,7 & 25,5 & 26,6 & 151,2 & 185,7 & 195,1 & 67 & 79 & 77 \\
Fev & 26,9 & 27 & 26,7 & 239,9 & 217,3 & 144,9 & 71 & 70 & 76 \\
Mar & 26,5 & 27,2 & 25,8 & 95,1 & 15,8 & 149,7 & 71 & 69 & 78 \\
Abr & 24 & 25,8 & 23,8 & 66,6 & 80,9 & 109,2 & 72 & 74 & 80 \\
Maio & 20,7 & 21,7 & 21,9 & 52,1 & 63,1 & 56,4 & 76 & 79 & 78 \\
Jun & 20,5 & 21,7 & 20,7 & 43,7 & 44,1 & 37,9 & 74 & 74 & 77 \\
Jul & 20,5 & 21 & 20,3 & 21,8 & 44,8 & 30,7 & 69 & 73 & 74 \\
Ago & 21,4 & 21,7 & 21,2 & 47,4 & 6 & 42,1 & 76 & 74 & 73 \\
Set & 22 & 23,9 & 21,9 & 65,3 & 17,5 & 62,3 & 77 & 69 & 75 \\
Out & 22,8 & 24,6 & 22,8 & 141 & 166,2 & 93,6 & 83 & 73 & 78 \\
Nov & 24 & 24,4 & 24 & 134,3 & 126,1 & 118,1 & 80 & 78 & 77 \\
Dez & 25,9 & 26,6 & 25,3 & 144,5 & 165,7 & 184,9 & 76 & 78 & 78 \\
\hline
\end{tabular}

os dois anos de estudo $\left(r_{\mathrm{s}}=0,79, p=0,003\right)$ e com as Normais climatológicas para o ano de $2006\left(r_{s}=0,89, p=0,0002\right)$. Não foi constatada correlação entre os dados do fator umidade relativa entre os anos e destes com os dados das Normais Climatológicas. Demonstra-se, portanto, pouca regularidade durante o período de coleta para este fator.

Quanto à sazonalidade das larvas de $D$. hominis em bovinos, ocorreu infestação ao longo dos 24 meses de estudo nas três propriedades (Tabela 2).

Tabela 2: Médias mensais de larvas de Dermatobia hominis em bovinos nas propriedades A, B e C nos municípios de Seropédica, Paracambi e Itaguaí, respectivamente, no período de janeiro de 2006 a dezembro de 2007

\begin{tabular}{|c|c|c|c|c|c|c|}
\hline \multirow{3}{*}{ Meses } & \multicolumn{6}{|c|}{ Ano } \\
\hline & \multicolumn{3}{|c|}{$\begin{array}{c}2006 \\
\text { Propriedades }\end{array}$} & \multicolumn{3}{|c|}{$\begin{array}{c}2007 \\
\text { Propriedades }\end{array}$} \\
\hline & $A$ & B & $\mathrm{C}$ & A & B & C \\
\hline Janeiro & 2,4 & 9,1 & 12,3 & 4,8 & 13,3 & 10,1 \\
\hline Fevereiro & 3,6 & 3,6 & 10,2 & 2,7 & 23,2 & 4,4 \\
\hline Março & 2,5 & 1,1 & 2,6 & 2,2 & 4,5 & 3,8 \\
\hline Abril & 2,7 & 3,2 & 0,8 & 2,9 & 1,4 & 6,6 \\
\hline Maio & 4 & 4,5 & 2,9 & 8,6 & 2,7 & 20 \\
\hline Junho & 9,4 & 2,9 & 8,6 & 0,7 & 7,7 & 5,3 \\
\hline Julho & 7,2 & 8,6 & 9,4 & 1,1 & 15,3 & 9,6 \\
\hline Agosto & 4 & 10,9 & 4,3 & 3 & 13,3 & 1,5 \\
\hline Setembro & 7,2 & 3,5 & 0,8 & 3,4 & 12,2 & 5,4 \\
\hline Outubro & 18,3 & 12 & 14,4 & 1,6 & 6,4 & 6,6 \\
\hline Novembro & 4,3 & 7,2 & 24,2 & 1,2 & 6,6 & 5,9 \\
\hline Dezembro & 8,1 & 5,9 & 25,8 & 2,6 & 10,5 & 10,5 \\
\hline
\end{tabular}

Na propriedade $A$ as contagens de bernes foram iguais entre o período de chuva e seca. Nas propriedades B e C a maior intensidade média de bernes foi registrada no período chuvoso. Entretanto, não diferiram significativamente das contagens da época seca. Por sua vez, em análise dos resultados de Souza et al. (2001), obtidos em estudo realizado nos mesmos municípios e em três outros, contata-se que apenas na propriedade do município de Paracambi a maior intensidade média de bernes foi registrada no período seco, entretanto sem diferença estatística significativa. Para as propriedades dos municípios de Japeri e Piraí, RJ, em que as maiores intensidade médias foram observadas no período chuvoso, também não houve diferença estatística significativa em relação ao período seco. Contudo, nas propriedades estudadas no município de Seropédica, Itaguaí, Mendes, RJ, as contagens do período chuvoso foram significativamente maiores, $p=0,05, p<0,01 \mathrm{e}$ $p<0,01$, respectivamente. Em estudo realizado em duas propriedades no município de Itaguaí, Carvalho (2002) constatou as maiores intensidade média no período chuvoso, mas sem diferença significativa com o período seco. Nos estudos realizados em municípios circunvizinhos aos do presente estudo, Vassouras e Engenheiro Paulo de Frontin, RJ, Maio et al. (2002a,b) também constataram as maiores intensidades médias de bernes no período chuvoso e com diferença significativa para o seco, $p=0,04$ e $p<0,01$, respectivamente.

O aumento de intensidade parasitária por berne em bovinos foi atribuído ao período chuvoso nos estudos de Horn e Antônio (1983), para as regiões Norte, Nordeste, Sudeste e Centro-Oeste do Brasil, de Maia e Guimarães (1985) e Magalhães e Lima (1988) para o estado de Minas Gerais, Souza et al. (2001) no Rio de Janeiro e de Pinto et al. (2002) em estudo em Palotina, no estado do Paraná.

Entretanto, em Uberaba, MG, Gomes e Maia (1988) constataram maiores intensidades parasitárias no período seco, porém, ao se comparar estes dados com os do período chuvoso, não houve diferença significativa. Por terem observado maiores intensidades de parasitismo no período seco, estes autores consideraram este fato como atípico ou influenciado pelos fatores biogeográficos. Resultado similar foi também observado por Silva Netto et al. (2001) em Rondônia.

Porém, destacam-se nos estudos de Horn e Antônio (1983) na região Sul do Brasil, Magalhães e Lesskiu (1982), Oliveira (1985) e Ribeiro et al. (1989), em Ponta Grossa, PR, Viamão e Pelotas, RS, respectivamente, a temperatura como o principal fator de influência e que as chuvas para a região são bem constantes (Oliveira, 1985) embora os meses chuvosos se manteham como os de maior ocorrência da dermatobiose.

Embora a análise de dados de outros estudos na região Sudeste e em outras regiões demonstre associação entre a intensidade de berne em bovinos e o período chuvoso, na análise de correlação entre a ocorrência de berne e as médias mensais dos fatores climáticos temperatura e precipitação não foi constatada diferença significativa em nenhum dos anos de avaliação, exceto na propriedade $A$, no ano de 2006, com os de umidade relativa (Tabela 3).

A ausência de correlação com a temperatura e precipitação também foi constatada nos dados referentes às contagens de bernes para os municípios de Japeri, Piraí, Seropédica e 
Tabela 3: Resultados das correlações de Spearman $\left(r_{s}\right)$ entre as médias mensais de larvas de Dermatobia hominis das propriedades dos municípios de Seropédica (A), Paracambi (B) e Itaguaí (C), estado do Rio de Janeiro, e os dados climáticos mensais, temperatura média compensada $\left({ }^{\circ} \mathrm{C}\right)$, precipitação pluvial total $(\mathrm{mm})$ e umidade relativa média (\%), dados da Estação Ecologia Agrícola SeropédicaRJ, dos anos de 2006 e 2007

\begin{tabular}{|c|c|c|c|c|c|c|c|c|c|c|}
\hline \multirow[b]{2}{*}{ Ano } & \multirow{2}{*}{$\begin{array}{c}\text { Test } \\
\mathrm{e}\end{array}$} & $A$ & $A$ & A & B & B & $B$ & C & C & C \\
\hline & & $\begin{array}{c}\mathrm{x} \\
\mathrm{T}\left({ }^{\circ} \mathrm{C}\right) \\
\end{array}$ & $\begin{array}{c}\mathrm{x} \\
\mathrm{P}(\mathrm{mm})\end{array}$ & $\begin{array}{c}x \\
\text { UR }(\%)\end{array}$ & $\begin{array}{c}x \\
T\left({ }^{\circ} \mathrm{C}\right) \\
\end{array}$ & $\begin{array}{c}\mathrm{x} \\
\mathrm{P}(\mathrm{mm})\end{array}$ & $\begin{array}{c}x \\
\text { UR }(\%)\end{array}$ & $\begin{array}{c}x \\
\mathrm{~T}\left({ }^{\circ} \mathrm{C}\right) \\
\end{array}$ & $\begin{array}{c}x \\
P(m m)\end{array}$ & $\begin{array}{c}x \\
U R(\%)\end{array}$ \\
\hline \multirow{2}{*}{2006} & $r_{s}$ & $-0,49$ & $-0,28$ & 0,63 & $-0,16$ & 0,13 & 0,25 & 0,25 & 0,51 & 0,18 \\
\hline & $p$ & 0,10 & 0,36 & 0,03 & 0,61 & 0,66 & 0,43 & 0,43 & 0,08 & 0,55 \\
\hline \multirow{2}{*}{2007} & $r_{s}$ & 0,09 & 0,04 & 0,28 & $-0,15$ & 0,15 & $-0,15$ & $-0,13$ & 0,46 & 0,57 \\
\hline & $p$ & 0,76 & 0,88 & 0,36 & 0,61 & 0,63 & 0,63 & 0,68 & 0,12 & 0,05 \\
\hline
\end{tabular}

Foi observada correlação positiva significativa em relação aos valores totais de dípteros coletados na propriedade B, com a precipitação pluvial $\left(r_{s}=0,80\right.$ e $\left.p=0,002\right)$ e na propriedade $\mathrm{C}$ com a temperatura $\left(r_{s}=0,60\right.$ e $\left.p=0,03\right)$, ambas no ano de 2007 (Tabela 8). Observaram-se também, correlações positivas e significativas, para os dados por famílias, entre os dípteros Muscidae e Calliphoridae com a preci-
Paracambi, no estudo de Souza et al. (2001). Também houve correlação entre a flutuação de bernes no local estudado por estes autores dentro do município de Seropédica com os dados de umidade relativa $\left(r_{s}=0,71\right.$ e $p<0,01)$. Porém, para os dados do município de Itaguaí foi constatada diferença significativa $\left(r_{s}=0,47 \mathrm{e}\right.$ $p=0,02)$ com a temperatura e com a precipitação $(r=0,6$ e $p<0,01)$. Não obstante, com os dados do estudo de Carvalho (2002) referentes a um ano de contagens de bernes em duas propriedades para este mesmo município, foi constatada diferença significativa com a precipitação $\left(r_{s}=0,63\right.$ e $\left.p=0,02\right)$ em uma propriedade e com a umidade relativa $\left(r_{s}=0,59\right.$ e $\left.p=0,04\right)$ para os dados da outra propriedade. Com os dados de Gomes e Maia (1988) em Uberaba também não foi constatada correlação com diferença estatística entre os dados de flutuação de bernes e os dados climáticos do período de estudo trabalhado por eles.

Outro importante elemento integrante do ciclo de $D$. hominis são as diferentes famílias de dípteros utilizadas por esta mosca para veicular seus ovos (Guimarães e Papavero, 1999). E cada família possui flutuações sazonais próprias e também sofrem influência dos fatores ambientais como os climáticos (Brito e Moya Borja, 2000).

No presente estudo, foi capturado um total de 18.966 dípteros, pertencentes a três principais famílias (Muscidae, Sarcophagidae e Calliphoridae). Destes $30,16 \%$ foram capturados na propriedade A, 34,59\% na propriedade B e $35,25 \%$ na propriedade C. Não houve diferença estatística significativa entre estes percentuais. Nas Tabelas 4 a 6 , estão os resultados agrupados por meses/ano/famílias/propriedades dos dípteros potenciais vetores capturados nas propriedades A, B e C, nos dois anos de estudo.

Não se constatou correlação significativa entre a média mensal de bernes e o total mensal de dípteros e nem pelo total mensal por família de dípteros capturados nas propriedades (Tabela 7). Resultado semelhante foi obtido quando foram confrontados os dados de dois estudos conduzidos em Uberaba, MG, um por Gomes e Maia (1988) que avaliaram a flutuação de bernes e outro de Maia e Gomes (1988) com o número total mensal de dípteros. Porém, entre os dois estudos supracitados, foi constatada correlação significativa $\left(r_{s}=0,61\right.$ e $\left.p=0,03\right)$ entre a flutuação de bernes e a flutuação de dípteros Sarcophagidae.
Tabela 4: Número de dípteros totais (DT) e por famílias - Muscidae (M), Sarcophagidae (S), Calliphoridae (C) - capturados na propriedade $A$, no município de Seropédica, RJ, nos anos de 2006 e 2007

\begin{tabular}{|c|c|c|c|c|c|c|c|c|}
\hline \multirow{3}{*}{ Meses } & \multicolumn{8}{|c|}{ Ano } \\
\hline & \multicolumn{4}{|c|}{2006} & \multicolumn{4}{|c|}{2007} \\
\hline & DT & $M$ & $S$ & C & DT & M & $\mathrm{S}$ & C \\
\hline Janeiro & 331 & 53 & 48 & 230 & 75 & 3 & 38 & 34 \\
\hline Fevereiro & 281 & 56 & 60 & 165 & 45 & 5 & 12 & 28 \\
\hline Março & 210 & 37 & 47 & 126 & 33 & 1 & 18 & 14 \\
\hline Abril & 151 & 25 & 11 & 115 & 105 & 1 & 58 & 46 \\
\hline Maio & 168 & 14 & 16 & 138 & 305 & 36 & 79 & 190 \\
\hline Junho & 128 & 3 & 53 & 72 & 153 & 20 & 50 & 83 \\
\hline Julho & 241 & 21 & 112 & 108 & 219 & 5 & 137 & 77 \\
\hline Agosto & 976 & 42 & 16 & 918 & 309 & 33 & 85 & 191 \\
\hline Setembro & 1147 & 37 & 4 & 1106 & 173 & 7 & 26 & 140 \\
\hline Outubro & 126 & 14 & 21 & 91 & 164 & 34 & 44 & 86 \\
\hline Novembro & 80 & 10 & 11 & 59 & 103 & 1 & 19 & 83 \\
\hline Dezembro & 40 & 5 & 2 & 33 & 157 & 9 & 42 & 106 \\
\hline
\end{tabular}

Tabela 5: Número de dípteros totais (DT) e por famílias - Muscidae (M), Sarcophagidae (S), Calliphoridae (C) - capturados em propriedade, no município de Paracambi, estado do Rio de Janeiro, nos anos de 2006 e 2007

\begin{tabular}{|c|c|c|c|c|c|c|c|c|}
\hline \multirow{3}{*}{ Meses } & \multicolumn{8}{|c|}{ Ano } \\
\hline & \multicolumn{4}{|c|}{2006} & \multicolumn{4}{|c|}{2007} \\
\hline & DT & $M$ & $\mathrm{~S}$ & C & DT & $M$ & $\mathrm{~S}$ & C \\
\hline Janeiro & 136 & 31 & 15 & 90 & 703 & 39 & 40 & 624 \\
\hline Fevereiro & 57 & 19 & 0 & 38 & 609 & 65 & 92 & 452 \\
\hline Março & 252 & 4 & 12 & 236 & 204 & 0 & 12 & 192 \\
\hline Abril & 212 & 11 & 58 & 143 & 225 & 9 & 49 & 167 \\
\hline Maio & 229 & 0 & 13 & 216 & 128 & 1 & 19 & 108 \\
\hline Junho & 43 & 2 & 22 & 19 & 135 & 0 & 28 & 107 \\
\hline Julho & 161 & 18 & 21 & 122 & 284 & 20 & 75 & 189 \\
\hline Agosto & 263 & 7 & 50 & 206 & 46 & 1 & 15 & 30 \\
\hline Setembro & 163 & 10 & 33 & 120 & 109 & 21 & 29 & 59 \\
\hline Outubro & 284 & 9 & 22 & 253 & 366 & 14 & 13 & 339 \\
\hline Novembro & 233 & 1 & 11 & 221 & 753 & 10 & 30 & 713 \\
\hline Dezembro & 209 & 7 & 47 & 155 & 756 & 130 & 16 & 610 \\
\hline
\end{tabular}

pitação da propriedade $\mathrm{B}$, no ano de $2007\left(r_{s}=0,67\right.$ e $\left.p=0,01\right)$ $\left(r_{s}=0,76\right.$ e $\left.p=0,004\right)$, respectivamente, e Sarcophagidae com os dados de temperatura, na propriedade $C$, em $2006\left(r_{s}=0,60\right.$ e $p=0,04)$. Registra-se que apenas três espécimes foram capturados portando ovos de $D$. hominis, dois Calliphoridae e um Sarcophagidae, na propriedade C em coleta realizada no mês de novembro de 2007. 
Tabela 6: Número de dípteros totais (DT) e por famílias - Muscidae (M), Sarcophagidae (S), Calliphoridae (C) - capturados em propriedade, no município de Itaguaí, estado do Rio de Janeiro, nos anos de 2006 e 2007

\begin{tabular}{|c|c|c|c|c|c|c|c|c|}
\hline \multirow{3}{*}{ Meses } & \multicolumn{8}{|c|}{ Ano } \\
\hline & \multicolumn{4}{|c|}{2006} & \multicolumn{4}{|c|}{2007} \\
\hline & DT & $M$ & $\mathrm{~S}$ & $\mathrm{C}$ & DT & $M$ & $\mathrm{~S}$ & C \\
\hline Janeiro & 117 & 35 & 69 & 13 & 717 & 26 & 35 & 656 \\
\hline Fevereiro & 137 & 16 & 92 & 29 & 843 & 106 & 79 & 658 \\
\hline Março & 118 & 3 & 67 & 48 & 586 & 13 & 32 & 541 \\
\hline Abril & 42 & 0 & 28 & 14 & 293 & 40 & 63 & 190 \\
\hline Maio & 263 & 45 & 42 & 176 & 412 & 17 & 27 & 368 \\
\hline Junho & 649 & 254 & 46 & 349 & 176 & 14 & 51 & 111 \\
\hline Julho & 301 & 45 & 46 & 210 & 258 & 7 & 45 & 206 \\
\hline Agosto & 127 & 48 & 17 & 62 & 66 & 10 & 23 & 33 \\
\hline Setembro & 131 & 23 & 65 & 43 & 12 & 2 & 1 & 9 \\
\hline Outubro & 58 & 4 & 26 & 28 & 10 & 1 & 2 & 7 \\
\hline Novembro & 212 & 18 & 76 & 118 & 364 & 53 & 30 & 281 \\
\hline Dezembro & 217 & 30 & 48 & 139 & 577 & 178 & 73 & 326 \\
\hline
\end{tabular}

O período relativamente longo, entre 80 e 150 dias, necessário para se completar o ciclo de $D$. hominis, a diversidade de espécies de dípteros que pode ser utilizada como vetores de seus ovos, e que a importância epidemiológica das espécies vetoras pode variar com a região, como destacado por Guimarães e Papavero (1999), torna complexa a associação entre a sazonalidade da dermatobiose em bovinos e a de seus vetores e destes com os fatores climáticos, principalmente, quando analisados pelas suas médias mensais ou por períodos quanto à intensidade de chuva.

Portanto, não é recomendado apontar isoladamente fatores como causas fundamentais à ocorrência do evento; e este, por ser complexo, multifatorial, torna-se necessário aprimorar as metodologias de coleta e análise dos dados para que seja alcançada melhoria no entendimento do fenômeno.

Tabela 7: Resultados das correlações para postos de Spearman $\left(r_{s}\right)$ entre as médias mensais de bernes (b) e os dípteros capturados por famílias - Muscidae (M), Sarcophagidae (S), Calliphoridae (C) - em propriedades nos municípios de Seropédica (A), Paracambi (B) e Itaguaí (C), estado do Rio de Janeiro, nos anos de 2006 e 2007

\begin{tabular}{|c|c|c|c|c|c|c|c|c|c|c|}
\hline \multirow{2}{*}{ Ano } & \multirow{2}{*}{ Teste } & \multicolumn{3}{|c|}{ Propriedade A } & \multicolumn{3}{|c|}{ Propriedade B } & \multicolumn{3}{|c|}{ Propriedade C } \\
\hline & & $b \times M$ & $b \times S$ & $b \times C$ & $b x M$ & $b \times S$ & $b \times C$ & $b \times M$ & $b x S$ & $b \times C$ \\
\hline 2006 & $r_{s}$ & $-0,71$ & $-0,13$ & $-0,52$ & 0,22 & 0,09 & 0,27 & 0,09 & 0,28 & 0,08 \\
\hline 2000 & $p$ & 0,01 & 0,66 & 0,07 & 0,48 & 0,78 & 0,37 & 0,76 & 0,37 & 0,78 \\
\hline 2007 & $r_{s}$ & 0,16 & 0,006 & 0,27 & 0,56 & 0,44 & 0,07 & 0,22 & 0,11 & 0,17 \\
\hline & $p$ & 0,60 & 0,99 & 0,39 & 0,05 & 0,15 & 0,81 & 0,48 & 0,73 & 0,58 \\
\hline
\end{tabular}

Tabela 8: Resultados das correlações de Spearman $\left(r_{s}\right)$ entre o total de dípteros em propriedades nos municípios de Seropédica (A), Paracambi (B) e Itaguaí (C), estado do Rio de Janeiro, e os dados climáticos mensais, temperatura média compensada $\left({ }^{\circ} \mathrm{C}\right)$, precipitação pluvial total $(\mathrm{mm})$ e umidade relativa média (\%), dados da Estação Ecologia Agrícola Seropédica, RJ, dos anos de 2006 e 2007

\begin{tabular}{|c|c|c|c|c|c|c|c|c|c|c|}
\hline \multirow{3}{*}{ Ano } & \multirow{3}{*}{ Teste } & \multicolumn{9}{|c|}{ Propriedades $\times$ Fatores Climáticos } \\
\hline & & \multicolumn{3}{|c|}{ Propriedade A } & \multicolumn{3}{|c|}{ Propriedade B } & \multicolumn{3}{|c|}{ Propriedade C } \\
\hline & & $\begin{array}{c}\mathrm{T} \\
\left({ }^{\circ} \mathrm{C}\right)\end{array}$ & $\begin{array}{c}\mathrm{P} \\
(\mathrm{mm})\end{array}$ & $\begin{array}{l}\text { UR } \\
(\%)\end{array}$ & $\begin{array}{c}\mathrm{T} \\
\left({ }^{\circ} \mathrm{C}\right)\end{array}$ & $\begin{array}{c}\mathrm{P} \\
(\mathrm{mm})\end{array}$ & $\begin{array}{l}\text { UR } \\
(\%)\end{array}$ & $\begin{array}{c}\mathrm{T} \\
\left({ }^{\circ} \mathrm{C}\right)\end{array}$ & $\begin{array}{c}\mathrm{P} \\
(\mathrm{mm})\end{array}$ & $\begin{array}{l}\text { UR } \\
(\%)\end{array}$ \\
\hline \multirow[t]{2}{*}{2006} & $r_{s}$ & $-0,08$ & $-0,16$ & $-0,37$ & 0,04 & $-0,01$ & 0,56 & $-0,47$ & $-0,44$ & $-0,03$ \\
\hline & $p$ & 0,80 & 0,60 & 0,22 & 0,88 & 0,97 & 0,05 & 0,12 & 0,14 & 0,90 \\
\hline \multirow{2}{*}{2007} & $r_{s}$ & $-0,76$ & $-0,41$ & 0,17 & 0,48 & 0,80 & 0,28 & 0,60 & 0,45 & 0,24 \\
\hline & $p$ & 0,005 & 0,18 & 0,58 & 0,10 & 0,002 & 0,36 & 0,03 & 0,14 & 0,43 \\
\hline
\end{tabular}

\section{Agradecimentos}

Ao apoio financeiro do CNPq e FAPERJ.

\section{Referências}

BRITO, L.G.; MOYA MORJA, G.E. Flutuação sazonal de Dermatobia hominis em peles bovinas oriundas de matadouro. Pesquisa Veterinária Brasileira, v. 20, n. 4, p. 151-154, 2000.
CARVALHO, C.R.P. Estudo da variação populacional de larvas de Dermatobia hominis (Linnaeus Jr., 1781) (Diptera: Cuterebridae) em bovinos e de dípteros veiculadores de seus ovos no município de Itaguaí, RJ. 2002. 68 f. (Dissertação)-Universidade Federal Rural do Rio de Janeiro, Seropédica, 2002. 
Departamento Nacional de Meteorologia. Normais Climatológicas (1961-1990). Brasília, DF, Ministério da Agricultura e Reforma Agrária, 1992. 84 p.

GOMES A.G.; MAIA, A.A.M. Berne: distribuição sazonal, localização no hospedeiro e susceptibilidade de bovinos mestiços na região de Uberaba, MG. Revista da Faculdade de Medicina Veterinária e Zootecnia, v. 25, n. 1, p. 109-115, 1988.

GUIMARÃES, J.H.; PAPAVERO, N. (ed.). Myiasis in man and animals in the Neotropical region. São Paulo: Plêiade/FAPESP, 1999, 308 p.

HORN, S.C.; ANTÔNIO, R.S. Carrapato, berne e bicheira no Brasil. Brasília, Ministério da Agricultura, 1983, 153 p.

MAGALHÃES, F.E.P.; LESSKIU, C. Efeito do controle do berne sobre o ganho de peso e qualidade dos couros em novilhos de corte. Pesquisa Agropecuária Brasileira, v. 17, n. 2, p. 329-336, 1982.

MAGALHÃES, F.E.P.; LIMA, J.D. Frequência de larvas de Dermatobia hominis (L. Jr.) em bovinos de Pedro Leopoldo, Minas Gerais. Arquivo Brasileiro de Medicina Veterinária e Zootecnia, v. 40, n. 5, p. 361 367, 1988.

MAIA, A.A.M.; GOMES, A.G. Vetores de Dermatobia hominis, Linnaeus Jr., 1781 (Diptera: Cuterebridae) na região de Uberaba, Minas Gerais. Revista da Faculdade de Medicina Veterinária e Zootecnia da Universidade de São Paulo, v. 25, n. 1, p. 47-51, 1988.

MAIA, A.A.M.; GUIMARÃES, M.P. Distribuição sazonal de larvas de Dermatobia hominis (Linnaeus Jr. 1781) (Diptera: Cuterebridae) em bovinos de corte na região de Governador Valadares, Minas Gerais. Arquivo Brasileiro de Medicina Veterinária e Zootecnia, v. 37, n. 5, p. 469-475, 1985.

MAIO, F.G.; SOUZA, W.M.; RAMALHO, L.D.; SANAVRIA, A.; FIGUEIREDO, M.A.; GRISI, L. Distribuição sazonal das larvas de Dermatobia hominis (Linnaeus Junior, 1781) em bovinos leiteiros no município de Vassouras, Rio de Janeiro, Brasil. Revista Brasileira de Ciência Veterinária, v. 9, n. 1, p. 29-31, 2002a.

MAIO, F.G.; RAMALHO, L.D.; SOUZA, W.M.; SANAVRIA, A.; FIGUEIREDO, M.A.; GRISI, L. Distribuição sazonal das larvas de Dermatobia hominis (Linnaeus Junior, 1781) em bovinos leiteiros no município de Engenheiro Paulo de Frontin, Rio de Janeiro, Brasil. Revista Scientia Vila Velha, v. 3, n. 1, p. 107-115, 2002b.
MOYA BORJA, G.E. Estudios sobre la biología, morfología y esterilización del tórsalo, Dermatobia hominis (L. Jr.). 1966. 63 f. (Tese)-IICA, Costa Rica, Turrialba, 1966.

OLIVEIRA, C.M.B. Variações mensais de infestações de bovinos por larvas de Dermatobia hominis (L. Jr.) em Viamão, RS. Arquivos da Faculdade de Veterinária da Universidade Federal do Rio Grande do Sul, v. 13, p. 61-64, 1985.

PINTO, S.B.; SOCCOL, V.T.; VENDRUSCOLO, E.; ROCHADELLI, R.; RIBEIRO, P.B.;FREITAG, A.; HENEMANN, C.; UEMURA, M. Bioecologia de Dermatobia hominis (Linnaeus Jr., 1781) em Palotina, Paraná, Brasil. Ciência Rural, v. 3, n. 5, p. 821-827, 2002.

RIBEIRO, P.B.;BRUM, J.G.V.W.; COSTA, P.R.P.;BATISTA, Z.R. Flutuação populacional de Dermatobia hominis (L. Jr., 1781) sobre bovinos no município de Pelotas, RS. Arquivo Brasileiro de Medicina Veterinária e Zootecnia, v. 41, n. 3, p. 223-231, 1989.

SERRA-FREIRE, N.M.; MELLO, R.P. (Ed.). Entomologia \& Acarologia na Medicina Veterinária. Rio de Janeiro: L. F. Livros, 2006, 200 p.

SILVA NETTO, F.G.; GOMES, A.; MAGALHÃES, J.A.; TAVARES, A.C. TEIXEIRA, C.A.D. 2001. Avaliação da avermectina no controle da mosca-do-berne (Dermatobia hominis) em Rondônia. CT/190, EMBRAPA-CPAF Rondônia, p. 2-6.

SOUZA, F.S.; FONSECA A.H.; PEREIRA, M.J.S. Validação da armadilha Adultrap ${ }^{\circledR}$ para captura de dípteros muscoides. Revista Brasileira de Parasitologia Veterinária, Jaboticabal, v. 18, n. 1, p. 66-68, 2009.

SOUZA, W.M.; BRITO, L.G.; MAIO F.G.; SANAVRIA, A. Estudo da variação sazonal de Dermatobia hominis (Linnaeus Jr., 1781) (Diptera: Cuterebridae) em diferentes regiões fisiográficas do estado do Rio de Janeiro. Revista Brasileira de Medicina Veterinária, v. 23, n. 1, p. 35-44, 2001. 